\title{
ACUMULACIÓN DE NUTRIENTES EN Stevia Rebaudiana (Bertoni)
}

\author{
ACCUMULATION OF NUTRIENTS IN Stevia Rebaudiana (Bertoni) \\ ACUMULAÇÃO DE NUTRIENTES EM Stevia Rebaudiana (Bertoni)
}

Olga B. Pérez Chamorro ${ }^{1}$

opchamorro@yahoo.com

Marcelo Calvache ${ }^{1}$

mcalvache20@gmail.com

\section{${ }^{1}$ Universidad Tecnológica Equinoccial Sede Santo Domingo, Ecuador}

Artículo recibido enero 2018, arbitrado febrero 2018 y publicado en mayo 2018

\section{RESUMEN}

Este estudio tuvo como finalidad determinar la acumulación total de macronutrientes en el cultivo de Stevia y la época de máxima absorción de nutrientes con fines de fertilización, para aumentar la sostenibilidad económica de medianos productores de la zona de Santo Domingo de los Tsáchilas. Fue una investigación experimental en Universidad Tecnológica Equinoccial, se usaron métodos como el de Kjeldhal y el $\mathrm{P}, \mathrm{K}, \mathrm{Ca} 2+, \mathrm{Mg} 2+, \mathrm{S}$ con el método de digestión húmeda con los ácidos nítrico y perclórico. Para finalizar Es importante tratar de fertilizar el cultivo de Stevia lo más fraccionado posible con los nutriente $\mathrm{N}, \mathrm{P}, \mathrm{K}$ y Mg, un tercio a la siembra, los 30 días y a los 50 días para cubrir la mayor demanda a los 60 y 90 días de edad.

Palabras clave: macronutrientes; sostenibilidad económica; cultivo de Stevia Rebaudiana

\section{ABSTRACT}

The purpose of this study was to determine the total accumulation of macronutrients in the Stevia crop and the period of maximum nutrient absorption for fertilization purposes, to increase the economic sustainability of medium-sized producers in the Santo Domingo of the Tsáchilas area. It was an experimental investigation at the Equinoctial Technological University, methods such as Kjeldhal and $\mathrm{P}, \mathrm{K}, \mathrm{Ca} 2+, \mathrm{Mg} 2+$, S were used with the method of wet digestion with nitric and perchloric acids. To finish it is important to try to fertilize the Stevia crop as fractionally as possible with the nutrients $\mathrm{N}$, $\mathrm{P}, \mathrm{K}$ and $\mathrm{Mg}$, one third at planting, 30 days and 50 days to cover the greater demand at 60 and 90 days old.

Key words: macronutrients; economic sustainability; Stevia Rebaudiana cultivation 
RESUMO

O objetivo deste estudo foi determinar o acúmulo total de macronutrientes na safra de estévia e o período de absorção máxima de nutrientes para fins de fertilização, para aumentar a sustentabilidade econômica de produtores de médio porte na área de Santo Domingo de o Tsáchilas. Foi uma investigação experimental na Universidade Tecnológica Equinocial, métodos como Kjeldhal e P, K, Ca2 ,$+ \mathrm{Mg} 2+, \mathrm{S}$ foram utilizados com o método de digestão úmida com ácidos nítrico e perclórico. Para finalizar É importante tentar fertilizar a safra de estévia o mais fracamente possível com os nutrientes $\mathrm{N}, \mathrm{P}, \mathrm{K}$ e $\mathrm{Mg}$, um terço no plantio, 30 dias e 50 dias para cobrir a maior demanda aos 60 e 90 dias. de idade.

\section{Palavras-chave:}

macronutrientes; sustentabilidade econômica; Cultivo de Stevia Rebaudiana

\section{INTRODUCCIÓN}

Stevia rebaudiana Bert., es una especie perenne de la familia de las Asteráceas, de origen paraguayo, utilizada por los nativos como edulcorante medicinal (Landázuri y Tigrero, 2009). Sus hojas tienen glucósidos dipertenos denominados "steviósidos" y "rebaudiósidos", cuyo poder edulcorante en extracto puro puede ser 300 veces mayor al de la sacarosa o azúcar de caña (Moreno, 2012). Además, los productos de esta especie no producen calorías, ventaja que se aprovecha para dejar de lado el consumo de edulcorantes artificiales nocivos para la salud (Bravo et al., 2009), característica que llama la atención de sectores agroindustriales que la consideran un sustituto de los endulzantes tradicionales y artificiales (Ramírez, et al., 2011).

El consumo de los productos de Stevia fue aprobada por la Administración de Alimentos y Fármacos (FDA) como edulcorante natural y seguro para el humano, al igual que la OMS y el Comité de Expertos en Aditivos Alimentarios de la FAO, quienes además, aprobaron su uso industrial (Alzate, 2012). En la actualidad, el mercado nacional e internacional tiende a utilizar cada vez más edulcorantes naturales por razones de conservación de la salud, especialmente para pacientes diabéticos y con obesidad (Goerzen y Salas, 2011); sólo en Ecuador, la diabetes mellitus es la primera causa de muerte, según estadísticas del INEC del 2011 (Ministerio de Salud Pública, 2013).

Dada su demanda, la producción mundial de esta especie se centra en productores como China, Paraguay Japón y Brasil, principales productores, y en menor escala, Corea, Canadá, Malasia, Brasil, Colombia, Ecuador, Argentina, Filipinas, Singapur, Tailandia, España, entre otros, con un promedio de 25000 ha cultivadas (REDIEX, 2010). En Ecuador, su cultivo está poco difundido y se siembran en extensiones menores de una hectárea en las provincias de Santo Domingo de los Tsáchilas, Pichincha, Manabí, Zamora, Francisco de Orellana, Loja, Sucumbíos y Carchi, y hasta de 15 ha en la provincia del Guayas (Landázuri y Tigrero, 2009). Datos de exportación registran que la compañía AgroeStevia exportó al mercado chileno y japonés $100000 \mathrm{~kg}$ a un precio de USD 3.50 en el año 2011 (Méndez y Méndez, 2012).

Aún se cultiva muy poca Stevia en Ecuador, a pesar de que la producción de cultivos extensivos hace vulnerable al productor, por las variaciones climáticas o de mercados, lo que hace necesario incentivar el cultivo de esta especie. Para lograr esto, un factor primordial es conocer el manejo agrícola, que en el país es poco conocido, igual que la fisiología relacionada con el crecimiento y rendimiento, especialmente en las condiciones agroclimáticas de la zona de 
Santo Domingo de los Tsáchilas. Un punto importante del manejo es el conocimiento de las necesidades nutricionales, absorción y transporte de nutrientes, y en el efecto de las limitaciones nutricionales de cada elemento sobre el crecimiento y desarrollo de la planta.

La presente investigación tuvo como finalidad determinar la acumulación total de macronutrientes en el cultivo de Stevia y la época de máxima absorción de nutrientes con fines de fertilización, para aumentar la sostenibilidad económica de medianos productores de la zona de Santo Domingo de los Tsáchilas, que cuenta con las condiciones climáticas favorables por la posición geográfica del país y que permite realizar hasta siete cortes al año (Méndez y Méndez, 2012).

\section{MATERIALES Y MÉTODOS}

El trabajo se desarrolló en el umbráculo experimental de la Universidad Tecnológica Equinoccial, sede Santo Domingo, ubicado en la Vía Chone $\mathrm{Km} 4$ 1/2. El estudio se efectuó durante el ciclo productivo de julio del 2013 a enero del 2014.

Geográficamente el sitio del experimento se ubicó a 79ㅇ 12' 34,3" de longitud oeste, $00^{\circ}$ 을 74' 1,7' de latitud sur y 504 msnm. La temperatura media mínima fue $20^{\circ} \mathrm{C}$, máxima $33^{\circ} \mathrm{C}$, y la humedad relativa de $57 \%$.

Los tratamientos consistieron en la fertilización completa con macronutrientes en dosis: $120,25,150,80,40$ y 55 partes por millón (ppm) de $\mathrm{N}, \mathrm{P}, \mathrm{K}, \mathrm{Ca}^{2+}, \mathrm{Mg}^{2+} \mathrm{y} \mathrm{S}$, respectivamente. En cuanto a micronutrientes se aplicaron en las siguientes dosis en ppm: $\mathrm{B}$, 1,21; $\mathrm{Cu}, 0,16$; Fe, 10,6; Mn, 1,20 y Zn²+, 0,6 (De Lima et al., 1997 b) (Pelayo, 2009).

Las plantas fueron evaluada en ocho observaciones: $15,30,45,60,75,90,105 \mathrm{y}$ 120 días después del trasplante (ddt) (De Lima, 2004; La unidad experimental consistió en una maceta plástica de $3 \mathrm{~L}$ llena con $3 \mathrm{~kg}$ de arena lavada, colocada sobre una tapa para recuperar y reutilizar el lixiviado, y sembrada con una planta de Stevia Para el enraizamiento se usaron esquejes terminales de 10 a $15 \mathrm{~cm}$ de largo, con cuatro a cinco nudos y cinco hojas abiertas y opuestas (Landázuri et al., 2009). Se aplicó el enraizador IBA $^{\mathrm{R}}$ 98\% PS (Acido 3-IndoleButirico) en el extremo inferior del esqueje al momento de plantarlo en la arena; sumergiéndolo por 10 segundos en una dosis de $3 \mathrm{~mL} \mathrm{~L}^{-1}$ de agua (Ramírez, 2011).

Posteriormente se plantaron 500 esquejes en bandejas germinadoras, usando como sustrato arena lavada y esterilizada a $120^{\circ} \mathrm{C}$ por 20 min (Ramírez et al., 2011;). A los 30 días de enraizamiento se trasplantaron los esquejes en las macetas con arena, previa desinfección de las raíces con Mancozeb 80\% en dosis de $2 \mathrm{~g} \mathrm{~L}^{-1}$. Las macetas fueron colocadas en mesas de concreto a $1 \mathrm{~m}$ de altura del suelo (Espitia, Montoya y Atencio, 2009).

La solución nutritiva madre se diluyó 1:10 con agua desionizada y se aplicaron a los dos días del trasplante, según los tratamientos sobre la arena y alrededor de las plantas. Los dos días sin aplicar la solución fueron para reponer las plantas muertas.

A cada unidad experimental se regó con agua desionizada llevando a capacidad de campo a las 18:00 horas, todos los días.

Para evaluar materia seca a cada planta se dividió en raíz, tallos y hojas; estos órganos por separado se secaron en una estufa a $70 \stackrel{\circ}{\circ}$ por 48 horas (Utumi et al., 1999). Se utilizó una balanza analítica para el pesaje y fundas de papel para separar los órganos en el secado. Para constatar las deficiencias de nutrientes se realizó un análisis foliar a cada unidad experimental en las siete evaluaciones realizadas; las hojas indicadoras fueron los últimos cinco pares de hojas extendidas de cada tallo (Utumi et al., 1999). El N se analizó 
con el método Kjeldhal y el $\mathrm{P}, \mathrm{K}, \mathrm{Ca}^{2}+\mathrm{Mg}^{2}+\mathrm{S}$ con el método de digestión húmeda con los ácidos nítrico y perclórico (Jácome y Sangurima, 2010; Álvarez y Marín, 2011). La Materia seca de la planta de Stevia fue la suma de los pesos secos de las hojas y los tallos (De Lima, 2004).

\section{RESULTADOS Y DISCUSIONES}

\section{Curva de crecimiento y fenología de la Stevia}

El contenido de materia seca o biomasa es una variable importante para el análisis del comportamiento del crecimiento de la planta y su estado fenológico (Fig1). La Stevia tuvo un continuo incremento de biomasa típico sigmoideo. Hasta los 30 días el crecimiento fue lento debido al estrés del trasplante, y de los 30 hasta los 90 días incrementó su tasa de desarrollo debido al aumento de hojas y tallos como fase vegetativa hasta los 60 días, donde inicia la etapa de floración y es recomendado realizar el corte para una producción comercial. Posteriormente al inició de su fase reproductiva registra el crecimiento acelerado y la mayor tasa de crecimiento absoluto hasta los 75 días. En esta etapa a pesar de que la materia seca aumenta, empieza a disminuir la tasa se crecimiento absoluto hasta la fructificación a los105 días.

Estos resultados fueron similares a los encontrados por Bonilla, Sánchez, y Perlaza (2008), donde se diferenciaron los siguientes periodos a los 71 días inició la floración, a los 115 días la fructificación, y diferentes de los encontrados por De Lima (2004), donde la floración inicia los 30 días y la fructificación a los 60 días, debido a la temperatura mayor que la de Santo Domingo de los Tsáchilas.

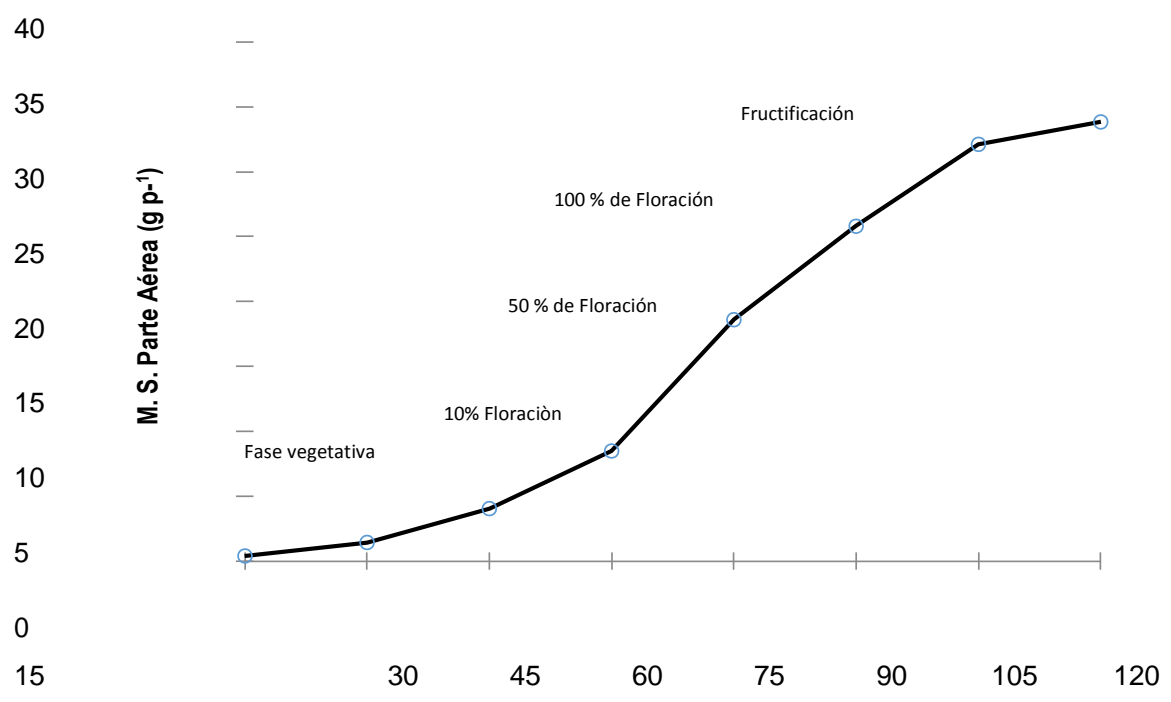

\section{Días después del trasplante (ddt)}

Figura 1. Curva de crecimiento de la parte aérea de la Stevia (tallo y hoja) 


\section{Distribución de la materia seca por órgano}

Días después del trasplante, se observó que la biomasa se localizó prioritariamente en hojas independiente de la edad de la plata, posteriormente en tallos con una proporción de 1 a 3 hasta los $45 \mathrm{ddt}, 1$ a 2 hasta los 75 días y 1:1 hasta los 120 ddt estos resultados son similares a los encontrados por De Lima (2004). En cuanto a la raíz la proporción es 1:4 hasta los 45 ddt y en adelante 1:2 hasta los 120 días.

\section{Extracción de nutrientes de la parte aérea de la Stevia evaluada hasta 120 ddt.}

Las extracción de los nutrimentos en las plantas de Stevia se elaboraron al relacionar el peso seco de la muestra (Cuadro 1) con la concentración de elementos de hojas y tallos, analizadas en las etapas de crecimiento. Considerando un cultivo comercial la extracción de nutrientes de tallo y hojas secas a los 60 ddt fue de 12.33; 1.61; 23.33; 23.43; 1.8 y $3.7 \mathrm{~kg} \mathrm{ha}^{-1}$ de $\mathrm{N}, \mathrm{P}, \mathrm{K}, \mathrm{Ca}^{2+}, \mathrm{Mg}^{2+}$ y $\mathrm{S}$, los mismos que comparados con los datos obtenidos por De Lima., et al (1997) a los 30 días e inicio de floración. 10.8; 1.3; 9.4; 2.6; 0.6 y $0.6 \mathrm{~kg} \mathrm{ha}^{-1}$ de N, P, K, $\mathrm{Ca}^{2+}, \mathrm{Mg}^{2+}$ y S, fueron ligeramente superiores definidos por el tiempo de extracción. Cuando es el caso de producir semillas la acumulación de materia seca es de $3384.75 \mathrm{~kg} \mathrm{ha}^{-1} \mathrm{y}$ de nutrientes es 62.16 ; 6.6; $80.8 ; 104.11 ; 15.15$ y $8.7 \mathrm{~kg} \mathrm{ha}^{-1} \mathrm{de}$ $\mathrm{N}, \mathrm{P}, \mathrm{K}, \mathrm{Ca}^{2+}, \mathrm{Mg}^{2+}$ y $\mathrm{S}$, se evidenció igual comportamiento.

Tabla 1. Extracción de Nutrientes $\mathrm{kg} \mathrm{ha}^{-1}$ en plantas de Stevia

\begin{tabular}{lllllllll}
\hline ddt & $\begin{array}{l}\text { \% MS. } \\
\text { Planta }^{-1}\end{array}$ & $\begin{array}{l}\text { MS. } \\
\mathrm{kg} \mathrm{ha}^{-1}\end{array}$ & $\begin{array}{l}\mathrm{N} . \\
\mathrm{kg} \mathrm{ha}^{-1}\end{array}$ & $\begin{array}{l}\mathrm{P} . \\
\mathrm{kg} \mathrm{ha}^{-1}\end{array}$ & $\begin{array}{l}\mathrm{K} . \\
\mathrm{kg} \mathrm{ha}^{-1}\end{array}$ & $\begin{array}{l}\mathrm{Ca}^{2+} . \\
\mathrm{kg} \mathrm{ha}^{-1}\end{array}$ & $\begin{array}{l}\mathrm{Mg.}^{2+} \\
\mathrm{kg} \mathrm{ha}^{-1}\end{array}$ & $\begin{array}{l}\mathrm{S} \\
\mathrm{kg} \mathrm{ha}^{-1}\end{array}$ \\
\hline 15 & 21.17 & 38.50 & 1.2 & 0.06 & 1.65 & 0.71 & 0.16 & 0.1 \\
30 & 17.56 & 143.25 & 3.46 & 0.41 & 4.84 & 5.27 & 0.43 & 0.3 \\
45 & 21.17 & 404.25 & 9.29 & 1.01 & 13.22 & 14.19 & 1.17 & 0.4 \\
$\mathbf{6 0}$ & $\mathbf{3 0 . 2}$ & $\mathbf{8 4 8 . 5 0}$ & $\mathbf{1 2 . 3 3}$ & $\mathbf{1 . 6 1}$ & $\mathbf{2 3 . 3 3}$ & $\mathbf{2 3 . 4 3}$ & $\mathbf{1 . 8}$ & $\mathbf{3 . 7}$ \\
75 & 29.88 & 1860.75 & 30.78 & 2.9 & 31.33 & 49.8 & 4.53 & 5.1 \\
90 & 37.92 & 2580.50 & 28.39 & 4.64 & 57.03 & 80.51 & 7.48 & 10.1 \\
105 & 32.61 & 3212.00 & 64.24 & 5.78 & 75.48 & 151.93 & 9.96 & 10.9 \\
120 & 62.24 & 3384.75 & 62.16 & 6.6 & 80.8 & 104.11 & 15.15 & 8.7 \\
\hline
\end{tabular}

\section{Curvas de extracción de nutrientes en función de la edad de la planta}

En la figura 2, se observa las curvas de extracción de nutrientes por la parte aérea que corresponde al modelo sigmoideo con coeficientes de correlación mayor a $\mathrm{R}^{2}=0.9$, que indica ajuste al modelo. La tasa de absorción más alta se registra entre los 60 y 90 días periodo en el cual no deben faltar los nutrimentos en la solución del suelo. Al obtener las derivadas de las ecuaciones se puede sacar la velocidad de absorción y obtener un perfecto exactamente cuál es la época de mayor extracción. 

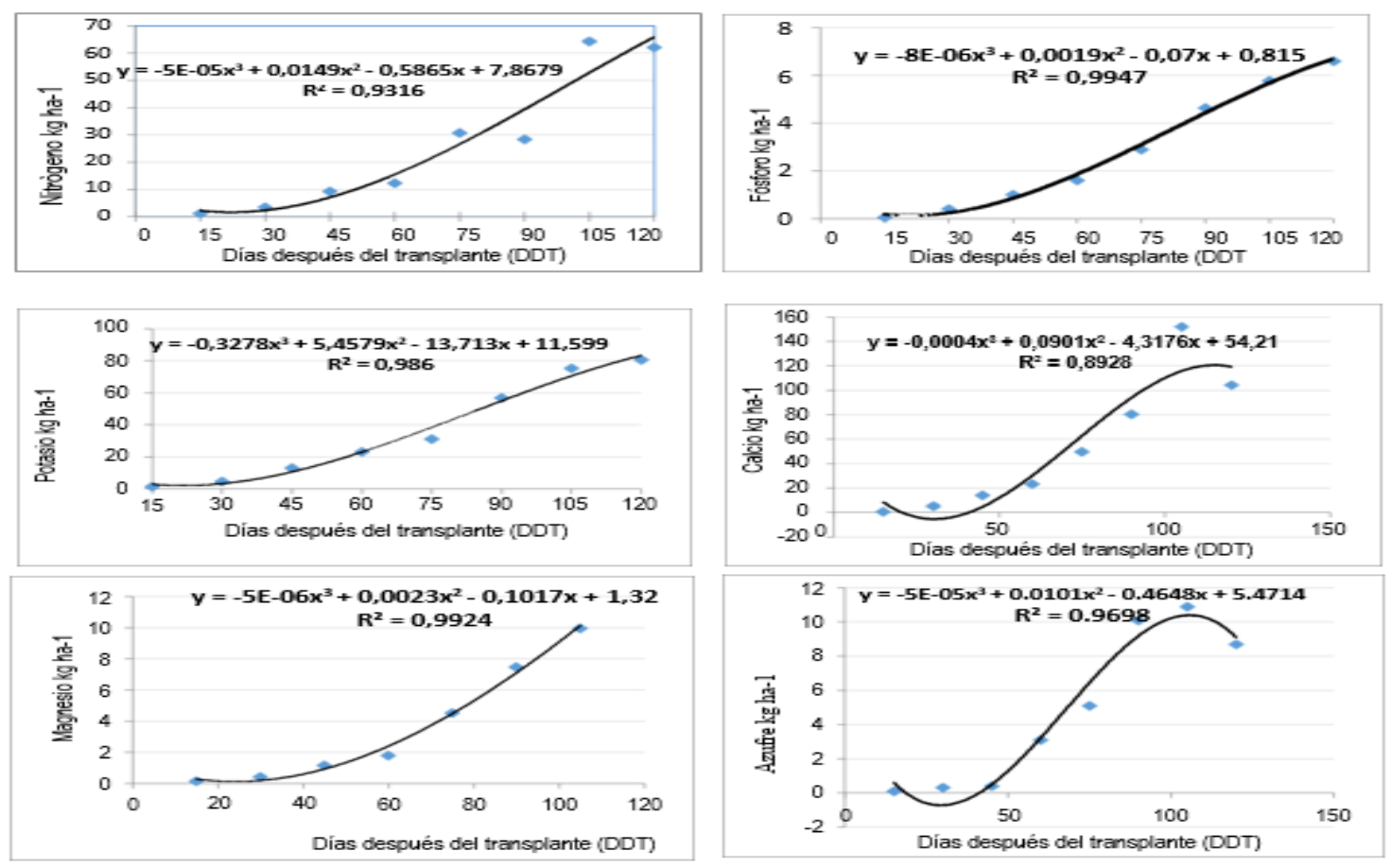

Figura 2. Curva de extracción de nutrientes en función de la edad de la planta hasta el final del ciclo.

En la tabla 2, se muestra la velocidad de absorción de nutrientes en kgha $^{-1}$ dìa $^{-1}$, obtenidas por derivadas parciales en las diferentes épocas de muestreo. La mayor extracción de $\mathrm{N}$ y $\mathrm{P}$ se registra entre los $60 \mathrm{a}$ 75 días, y entre los 75 a 90 días para el $\mathrm{K}, \mathrm{Ca}^{2+}$, $\mathrm{Mg}^{2+}$ y S. Cuando se cultiva con la finalidad de obtener semillas entre los 105 a 120 días la absorción es prácticamente nula no se debería aplicar nutrientes. Estos resultados tuvieron similitud con los datos obtenidos por Goettemoeller y Ching, citados en Sumida (1980), donde el período de mayor absorción de nutrientes encontrado fueron entre 60 a 90 días después del trasplante al campo.

Tabla 2. Velocidad de Absorción de Nutrientes kg ha- ${ }^{1}$ día $^{1}$

\begin{tabular}{llllllll}
\hline DDT & $\mathbf{M S}$ & $\mathbf{N}$ & $\mathbf{P}$ & $\mathbf{K}$ & $\mathbf{C a}^{\mathbf{2 +}}$ & $\mathbf{M g}$ & $\mathbf{S}$ \\
\hline Kg ha- $^{\mathbf{1}}$ día $^{\mathbf{1}}$ & & & & & & & \\
$\mathbf{1 5 - 3 0}$ & 6,98 & 0,15 & 0,02 & 0,21 & 0,30 & 0,02 & 0,02 \\
$\mathbf{3 0 - 4 5}$ & 17,40 & 0,39 & 0,04 & 0,56 & 0,59 & 0,05 & 0,04 \\
$\mathbf{6 0 - 7 5}$ & $\mathbf{6 7 , 4 8}$ & $\mathbf{1 , 4 1}$ & $\mathbf{0 , 0 9}$ & 0,53 & 1,76 & 0,18 & 0,09 \\
$\mathbf{7 5 - 9 0}$ & 47,98 & 0,86 & 0,08 & $\mathbf{1 , 7 1}$ & $\mathbf{2 , 0 5}$ & $\mathbf{0 , 2 0}$ & $\mathbf{0 , 1 2}$ \\
$\mathbf{9 0 - 1 0 5}$ & 42,10 & 0,54 & 0,07 & 1,23 & 0,84 & 0,17 & 0,08 \\
$\mathbf{1 0 5 - 1 2 0}$ & 11,52 & 0,50 & $-0,004$ & 0,35 & 0,73 & 0,01 & 0,05 \\
\hline
\end{tabular}




\section{CONCLUSIONES}

La acumulación total de nutrientes hasta los 60 días fue de de $12.33 ; 1.61 ; 23.33 ; 23.43$; 1.8 y $3.7 \mathrm{~kg} \mathrm{ha}^{-1}$ de $\mathrm{N}, \mathrm{P}, \mathrm{K}, \mathrm{Ca}^{2+}, \mathrm{Mg}^{2+}$ y S respectivamente. $Y$ hasta los 120 días 62.16; 6.6; 80.8; $104.11 ; 15.15$ y 8.7.6 $\mathrm{kg} \mathrm{ha}^{-1}$ de N, P, $\mathrm{K}, \mathrm{Ca}^{2+}, \mathrm{Mg}^{2+}$ y $\mathrm{S}$ respectivamente.

La máxima tasa de extracción de nutrientes se da a los 60 día para N y P 1.41, 0.09 kgha $^{-1} \mathrm{dìa}^{-1} \mathrm{y}$ a los 75 para $\mathrm{K}, \mathrm{Ca}^{2+} \mathrm{Mg}^{2+} \mathrm{y}$ $\mathrm{S}$ con $1.71 ; 2.05 ; 0.20 ; 0.12$ kgha-1 $^{-1}$ ìa

Es importante tratar de fertilizar el cultivo de Stevia lo más fraccionado posible con los nutriente $\mathrm{N}, \mathrm{P}, \mathrm{K}$ y $\mathrm{Mg}$, un tercio a la siembra, los 30 días y a los 50 días para cubrir la mayor demanda a los 60 y 90 días de edad.

\section{REFERENCIAS}

Alzate, J. (2012). Producción y comercialización de Stevia en el municipio de Ibague Vereda Potrerito. Universidad de Tolima

Bonilla, C. R., Sánchez, M. S., y Perlaza, D. F. (2008). Evaluación de métodos de propagación, fertilización nitrogenada y fenología de Stevia en condiciones del Valle del Cauca. Acta Agronómica, 56(3). Recuperado el 15 de agosto del 2012, de http://www.redalyc.org/articulo.oa?id=1 69913315004

Bravo, M., Ale, N., Rivera, D., Huamán, j., Delmás, D., Rodríguez, M., Bautista, M. (2009). Caracterización química de la Stevia Rebaudiana. Química, 12(2), 5-8. Recuperado el 12 de agosto DEL 2012 http://www.revistasinvestigacion.unms m.edu.pe/index.php/quim/article/view/ 4292

De Lima F. (2004). Análise Quantitativa do

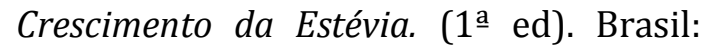
Embrapa Agropecuária

De Lima F., y Malvolta, E. (1997). Síntomas de desórdenes nutricionales en eStevia Stevia rebaudiana (Bert.). Scientia Agrícola (54) (1-2) 53-51. Recuperado el 13 de junio del 2014, de http://www.scielo.br/scielo.php?script=s ci_arttextypid=S0103-
$90161997000100008 y \operatorname{lng}=$ enytlng=.\%20 10.1590/S0103-90161997000100008

De Lima, F., Malavolta, E., De Sena, O., y. Carneiro.J. (1997). 1997b. Absorção e acumulação de nutrientes em estévia Stevia rebaudiana (Bert.) Bertoni: II. Micronutrientes. Sci. agric. 54(1-2) Espíndola, C. (2004). Prácticas de biología de organismos multicelulares. Bogotá: Pontificia Universidad Javeiana

Espitia, C., Montoya, R. y Atencio L. S. (2009). Rendimiento de Stevia rebaudiana Bert. Bajo tres arreglos poblacionales en el Sinú Medio. Actualidad y Divulgación Científica 12 (1):151-168. Recuperado el 18 de mayo del 2017, de http://www.scielo.org.co/scielo.php?scri pt=sci_arttextypid=S0123-

42262009000100016

Goerzen, H., y Salas, D. (2011). El sorprendente legado que nos dejó el sabio Moisés Bertoni: el Ka'a He`e. Ciencia y Salud, (9), 60-63. Recuperado el 05/05/2014, de http://scielo.iics.una.py/pdf/iics/v9n1/v 9n1a09.pdf

Landázuri, P., y Tigrero J. (2009). Generalidades. In P.A. Landázuri A., y J.O. Tigrero S. (eds.), Stevia rebaudiana Bertoni, una planta medicinal. $1^{\underline{a}}$ ed. EDIESPE, Sangolquí - Ecuador. p. 1- 7

Méndez, A., y Méndez, H. (2012). Nuevos mercados para hojas de exportación en Stevia: Propuesta estratégica de comercialización, caso Stevia peninsular. "Tesis de maestría no publicada", Universidad Politécnica Salesiana, Santa Elena, Ecuador

Moreno, A. (2012). Análisis de la incidencia y relación de los factores ambientales en el desarrollo vegetativo de la Stevia Rebaudiana $B$. en condiciones de Invernadero en la Sabana de Bogotá. Tesis de maestría en gestión de procesos. Universidad de la Sabana, Bogotá. Recuperado 05 de mayo del 2017, de http://intellectum.unisabana.edu.co:8080/j spui/bitstream/10818/8324/3/ALEJANDR O\%20MO RENO\%20tesis\%20FINAL.pdf 
MSP Ministerio de Salud Pública. (14 de Noviembre de 2013). Día mundial de la Diabetes: MSP ejecuta acciones para reducir su incidencia y complicaciones. Quito, Pichincha, Ecuador. Recuperado el 20 de mayo del 2017, de http://www.salud.gob.ec/dia-mundialde-la-diabetes- msp-ejecuta-accionespara-reducir-su-incidencia-ycomplicaciones/

Pelayo B. (ed.). (2009). Manual de prácticas de fisiología vegetal. Universidad Autónoma de Ciudad Juárez, Chihuahua, México. Recuperado el 15 de septiembre del 2012, de http://sirio.uacj.mx/ICB/cqb/licenciatur aenbiolog\%C3\%ADa/Documents/Manual es/intermedi o/FISIOLOGIA\%20VEGETAL.pdf

Ramírez, J., Avilés B., Moguel O., Góngora G., y C. May, L. (2011). Stevia (Stevia Rebaudiana, Bertoni), un cultivo con potencial productivo en México. (1aㅡ ed).
Centro de Investigación Regional Sureste, Mérida, México

REDIEX. (2010). Perfiles de productos para la exportación. Asunción, Paraguay. Recuperado el 15 de mayo del 2017, de http://www.rediex.gov.py/userfiles/file/ 44\%20-\%20PPE\%20Stevia .pdf.

Sumida, T. (1980). Studies on S. Rebaudiana as a new possible crop for sweetening resource in Japan J. Central Ag. Exp. Stn. 31:1-71

Utumi, M.M., Monnerat, P. H., Gomes, P.R., Rezende, P.C., y Campos, G. (1999). Deficiência de macronutrientes em estévia: sintomas visuais e efeitos no crescimento, composição química e produção de esteviosídeo. Pesq. agropec. bras. 34(6):1039-1043. Recuperado 15 de Septiembre del 2017, de http://www.scielo.br/scielo.php?script=s ci_arttextypid=S0100204X1999000600016 